\title{
Gauging Possibilities for Action Based on Friction Underfoot
}

\author{
Amy S. Joh, Karen E. Adolph, Priya J. Narayanan, and Victoria A. Dietz \\ New York University
}

\begin{abstract}
Standing and walking generate information about friction underfoot. Five experiments examined whether walkers use such perceptual information for prospective control of locomotion. In particular, do walkers integrate information about friction underfoot with visual cues for sloping ground ahead to make adaptive locomotor decisions? Participants stood on low-, medium-, and high-friction surfaces on a flat platform and made perceptual judgments for possibilities for locomotion over upcoming slopes. Perceptual judgments did not match locomotor abilities: Participants tended to overestimate their abilities on low-friction slopes and underestimate on high-friction slopes (Experiments 1-4). Accuracy improved only for judgments made while participants were in direct contact with the slope (Experiment 5), highlighting the difficulty of incorporating information about friction underfoot into a plan for future actions.
\end{abstract}

Keywords: prospective control, perception-action, friction, slopes, locomotion

\section{Prospective Control}

A basic tenet of the perception-action approach is the coupling between perceptual information and the adaptive control of movement (E. J. Gibson \& Pick, 2000; J. J. Gibson, 1979). Perceptual information allows prospective control of action through a feedforward mechanism in which current movements generate perceptual information for detecting potential problems, planning future actions, and executing actions appropriately (Patla, 1991, 1997b, 1998). Exploratory movements, such as peering with the eyes and rubbing the feet over the ground surface, are performed with the explicit purpose of generating and gathering relevant information for planning future actions. Every movement, however, whether intentional or not, provides fodder for the perceptual system.

Prospective control is especially important for guiding locomotion adaptively. In contrast to the small movements involved in looking and reaching, the body undergoes large displacements during locomotion, and missteps can have serious consequences for safety. Exploratory movements are crucial because detecting problems and planning future actions require perceptual information from a distance (Patla, 1991, 1997b). To prepare for a slippery patch of ground, for example, walkers need a few steps to dampen the forces to brace themselves for contact or to redirect forces to

Amy S. Joh, Karen E. Adolph, Priya J. Narayanan, and Victoria A. Dietz, Department of Psychology, New York University.

This research was supported by NICHHD Grant 33486 awarded to Karen E. Adolph and New York University Dean's Undergraduate Research Fund Grants awarded to Priya J. Narayanan and Victoria A. Dietz. We are grateful to the members of the New York University Infant Action Lab for assistance with data collection, Allen Mincer for lessons on friction, and David Dietz for the new walkway. We thank Claire Michaels, Bill Warren, and Tom Stoffregen for their helpful comments and suggestions on drafts of this article.

Correspondence concerning this article should be addressed to Amy S. Joh, Department of Psychology, New York University, 4 Washington Place, Room 416B, New York, NY 10003. E-mail: aj394@nyu.edu veer around it (Patla, 1989; Patla, Prentice, Robinson, \& Neufeld, 1991; Patla, Robinson, Samways, \& Armstrong, 1989). Without sufficient warning, walkers are unlikely to generate intentional exploratory movements, and prospective control may be disrupted. Reactive adjustments are only a method of last resort. An illplanned step on icy ground, for example, may result in a fall. The problem of maintaining balance is further exacerbated when the ground is uneven or slanting. On slippery slopes, carefully planned and executed actions are essential for keeping balance.

Prospective control is supported by multiple sources of perceptual information about the layout of the environment, the status of walkers' bodies, and the relationship between the two. For example, an assortment of visual depth cues - convergence, stereopsis, texture gradients, motion parallax, eye height, and so on-specify structural variations in the environmental layout such as surface slant, obstacles in the path, and changes in elevation (Fajen \& Warren, 2003; Kinsella-Shaw, Shaw, \& Turvey, 1992; Mark, Baillet, Craver, Douglas, \& Fox, 1990; Mark, Jiang, King, \& Paasche, 1999; Proffitt, Creem, \& Zosh, 2001; Warren, 1984; Warren \& Whang, 1987). Reciprocally, vestibular and optic flow information specify the current status of walkers' balance, body dimensions, and time to contact an obstacle (Fajen \& Warren, 2003; Lee \& Aronson, 1974; Lee, Lishman, \& Thomson, 1982; Stoffregen, 1985; Warren, Young, \& Lee, 1986). Using these cues as a basis for prospective control, walkers show remarkable abilities to gauge possibilities for action under varying and novel environmental conditions, such as estimating the steepest navigable slope (e.g., Fitzpatrick, Carello, Schmidt, \& Corey, 1994; Kinsella-Shaw et al., 1992; Proffitt, Bhalla, Gossweiler, \& Midgett, 1995; Proffitt et al., 2001). Subtle, barely discernable exploratory movements of the head and body can produce sufficient perceptual information for adaptive locomotor decisions (Warren \& Whang, 1987).

\section{Friction Underfoot}

In addition to visual information for the ground surface ahead and movements of the body through space, walkers' movements 
generate tactile information at the feet that can specify the current status of the self vis-à-vis the environment. Information about friction is especially important for locomotion because it is ubiquitous. With every step, shuffle, or sway, walkers can feel their feet slip or catch against the ground surface. Under continuous ground conditions, such as an ice-covered sidewalk or wall-to-wall carpeting, current forces at the feet provide information for controlling future actions.

In contrast to the rich literature on walkers' use of visual information for guiding balance and locomotion prospectively, there has been little work focused on walkers' use of tactile information underfoot (Cohen \& Cohen, 1994a, 1994b). One exception is Adolph and colleagues' studies of infants' ability to use information about friction underfoot prospectively (Adolph, Eppler, \& Joh, 2007; Lo, Avolio, Massop, \& Adolph, 1999). Fourteen-month-old walking infants stood on a flat starting platform facing an adjustable sloping walkway $\left(0^{\circ}-90^{\circ}\right)$. Both the flat and sloping sections were covered with the same low-friction vinyl or high-friction rubber surfaces. The low-friction condition was so treacherous that infants could not walk down slopes steeper than $6^{\circ}$. (Indeed, several infants were excluded from the study because they could not walk over $0^{\circ}$ without slipping.) In contrast, the high-friction condition allowed infants to walk down slopes steeper than $15^{\circ}$. Although previous work showed that 14-montholds match their attempts to walk to the conditional probability of success, the earlier experiments could not assess whether infants relied on both visual cues about the layout and friction information at their feet (Adolph, 1995; Adolph \& Avolio, 2000; Adolph, Eppler, \& Gibson, 1993; Eppler, Adolph, \& Weiner, 1996). Thus, if infants incorporate tactile information about friction with other sources of perceptual information, then they should have avoided very shallow slopes when the walkway was covered with the low-friction surface and attempted to walk down steeper slopes when the walkway was covered with the high-friction rubber. However, they did not. Infants attempted to walk down comparably steep slopes in both conditions, suggesting that they did not incorporate tactile information about friction underfoot with visual cues for the slope of upcoming ground.

Two possible explanations for infants' failure to use underfoot information about friction can be eliminated. First, the problem was not insensitivity to the feeling of slip: On the slippery vinyl, infants adjusted their gait patterns to walk over the flat starting platform toward the slope. Under low-friction conditions, small, slow, stiffly upright steps maximize the size of the normal forces (Cham \& Redfern, 2002; Marigold \& Patla, 2002; Myung \& Smith, 1997; You, Chou, Lin, \& Su, 2001). Although sensitivity to information for friction is clearly necessary, it may not be sufficient for guiding actions adaptively. Second, the problem was not an inability to explore the friction conditions. While approaching steep slopes in both friction conditions, infants performed exploratory movements at the brink (e.g., rocking around the ankles or rubbing the feet against the slope). However, only the sight of a steep slope, not the feeling of slip underfoot, elicited such deliberate tactile exploration.

A third potential reason for infants' failure to integrate the forces they feel beneath their feet with the available visual information about upcoming variations in surface slant may be the complicated nature of friction. The common sense view that friction is a property of the ground surface (e.g., ice is slippery, concrete is not) is inaccurate. Friction is a resistive force that emerges only when two surfaces come into contact with each other. Everyday activities, such as walking, are impossible without sufficient resistive forces. The amount of frictional force required for everyday locomotion depends on the materials covering the sole of the shoe and the ground, the angle of walkers' bodies, the wear and tear of walkers' shoe soles, the presence of contaminants on the floor, and so on. Thus, ice is slippery only because of the particular type of shoe sole that landed on it and because of the manner in which the step occurred.

Sloping ground exacerbates low-friction conditions. To prevent an object from slipping down a slope, frictional force must be strong enough to overcome the pull of gravity. The amount of frictional force that is created depends on the coefficient of friction (COF) between the object and the surface of the slope. Thus, as the slope increases, the gravitational pull also increases, requiring larger amounts of frictional force-and higher COF between the object and the surface-to prevent slipping. On a $90^{\circ}$ slope, gravity will overtake friction and the object will fall regardless of the COF. Between $0^{\circ}$ and $90^{\circ}$, the point at which gravity becomes larger than the frictional forces and causes an object to slip depends on the COF between the slope's surface and the object.

As such, friction presents a nonlinear and variable problem for walkers. For example, the same increase in slant may become direr on steeper slopes. Failing to detect the potential for slipping, not planning the appropriate methods for walking, and deciding to attempt walking when the potential for falling is uncertain may be riskier maneuvers when slant increases from $30^{\circ}$ to $35^{\circ}$ than when it increases from $0^{\circ}$ to $5^{\circ}$. Moreover, the base of support decreases on steeper slopes because the feet are at a greater angle so that fighting the pull of gravity becomes more difficult (Adolph \& Avolio, 2000).

\section{Current Aims}

In five experiments, we examined the role of underfoot information about friction in walkers' decisions for locomotion over upcoming ground. Participants judged possibilities for coping with slopes by extrapolating from the friction conditions they felt under their feet. In Experiment 1, an adjustable slope was covered with low-, medium-, and high-friction materials, and participants judged the steepest slopes that they thought they could navigate without slipping or falling. In Experiment 2, participants made large exploratory movements before each decision to gather additional perceptual information. In Experiment 3, participants made judgments about walking uphill, walking downhill, and standing on slopes while wearing shoes with low-, medium-, and highfriction soles. In Experiments 1-3, as a measure of accuracy, participants' perceptual judgments were compared to lead block slip thresholds obtained by placing a lead block on a slope and finding the steepest slope at which the block began sliding over each of the surfaces.

In Experiments 4 and 5, again participants judged their ability to cope with low-, medium-, and high-friction slopes. However, we tested the accuracy of participants' perceptual judgments by directly comparing their perceived and actual abilities. After making perceptual judgments, participants stood on slopes covered with the different surfaces to determine their standing slip thresholds. Moreover, in Experiment 5, participants judged slopes while 
touching the brink with one foot to obtain additional tactile information about friction and slant.

We reasoned that if participants' perception of their abilities changed with friction conditions and matched the slip thresholds, then these experiments would provide evidence that adult walkers extrapolate from flat to upcoming sloping ground based on information about friction underfoot, and that they may use information about friction to plan their actions prospectively. However, if adults fared more like infants and did not show a match between perceptual judgments and abilities, then the results would suggest that integrating friction underfoot with other sources of perceptual information for prospective control is difficult for adult walkers despite greater age and experience.

\section{Experiment 1: Judging the Steepest Slope for Safe Walking}

One way to investigate whether walkers incorporate tactile information about friction beneath their feet with visual information about the ground ahead is to require them to judge what may be possible in the future based on what they are feeling now. While standing on flat, low-friction, medium-friction, and high-friction surfaces, participants reported the steepest slope that they perceived they could walk down without slipping or falling. For all friction conditions, the standing platform and slope were covered with the same materials. The task was a partial replication of previous studies with infants (Adolph, 1995; Adolph \& Avolio, 2000; Adolph et al., 2007; Lo et al., 1999); the surface materials were identical to those used for testing infants. However, because the sloping walkway was designed to hold infants and could not accommodate adults' height or weight, we could not obtain measurements of participants' actual performance. Instead, perceptual judgments were tested against lead block slip thresholds as an estimate of accuracy.

\section{Method}

Participants. Twenty-four adults ( 15 women, 9 men; $M$ age $=$ 20.33 years, $S D=1.73$ ) participated in exchange for course credit or monetary compensation. Only 1 had experienced a frictionrelated fall requiring medical care. Data from 2 additional adults were excluded from the final sample due to equipment failure.

Apparatus. As illustrated in Figure 1A, participants stood on a standing platform $(76.4 \mathrm{~cm}$ wide $\times 54.4 \mathrm{~cm}$ long $\times 18-54 \mathrm{~cm}$ high) facing an adjustable sloping walkway $\left(0^{\circ}-90^{\circ}\right)$. The height of the standing platform varied in $18-\mathrm{cm}$ increments so that the top of participants' heads was approximately $50 \mathrm{~cm}$ from the ceiling (Figures 1A-1C). The sloping walkway was composed of two flat sections flanking a middle sloping section (each $86 \mathrm{~cm}$ wide $\times 92$ $\mathrm{cm}$ long) that participants adjusted via a hand-held remote. Protractors on the sides of the walkway out of participants' sight indicated the angle of slant to the experimenter. The viewing platform and sloping walkway were covered with low-friction vinyl Naugahyde upholstery material, medium-friction plush carpet, or high-friction rubber Dycem (a tacky nonslip material used widely in medicine and therapy, e.g., on wheelchairs and footrests), so that participants were standing on and looking at identical surfaces. The surface materials were stretched flat and glued to wooden backings. All of the surfaces were blue. We selected the
A. Standing Platform and Sloping Walkway (Two Blocks)

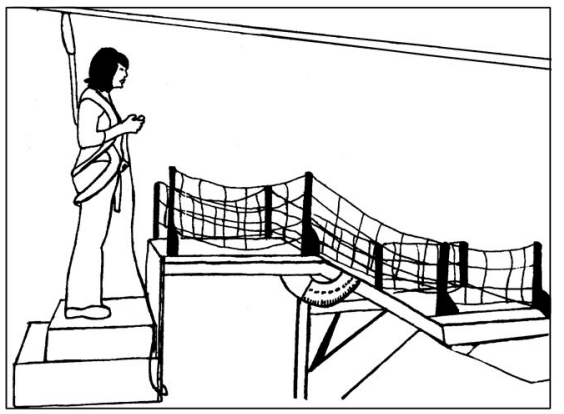

B. Three Blocks

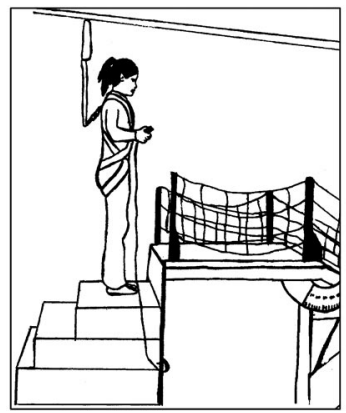

C. One Block

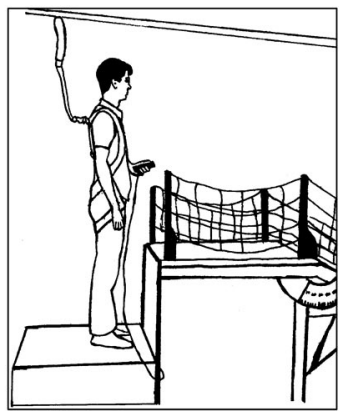

Figure 1. (A) In Experiments 1-3, participants made perceptual judgments from a standing platform while looking down the sloping walkway. The height of the standing platform was adjusted to ensure a similar view of the sloping walkway for all participants. Participants between $166 \mathrm{~cm}$ and $182 \mathrm{~cm}$ in height stood on two blocks, (B) participants shorter than 166 $\mathrm{cm}$ stood on three blocks, and (C) participants taller than $182 \mathrm{~cm}$ stood on one block.

vinyl and rubber surfaces to be relatively novel but similar in color and texture so that participants would be encouraged to focus on tactile and depth cues rather than to rely on familiar associations.

Participants wore nylon tights so that the COF at the foot-floor interface remained similar across participants. We obtained lead block slip thresholds for each nylon-surface pairing by placing a nylon-covered lead block (4,330 g) on each surface. By slowly increasing the slant of the slope from $0^{\circ}$, we determined the lead block slip threshold: the shallowest angle that caused the block to slide down the slope continuously. For reliability, slip threshold trials were repeated 10 times on each surface. Lead block slip thresholds were $16.90^{\circ}$ for vinyl $(S D=.99), 30.96^{\circ}$ for carpet $(S D=2.06)$, and $50.80^{\circ}$ for rubber $(S D=3.58)$ (see Table 1$)$.

Procedure. For safety, participants wore a full-body harness connected to a shock-absorbing 111-cm-long cable lanyard attached to ceiling struts directly above the viewing platform. The rigging was loose enough for participants to move freely. An experimenter stood near participants on all trials to provide assistance if necessary.

Participants reported four judgments for each of the three surfaces (vinyl, carpet, and rubber) for a total of 12 trials. For each surface, 2 trials began with the slope horizontal at $0^{\circ}$ and 2 with the slope vertical at $90^{\circ}$. All sessions began with medium-friction carpet trials so that participants could become familiar with the 
Table 1

Experiments 1 and 2: Lead Block Slip Thresholds and Perceptual Judgments

\begin{tabular}{|c|c|c|c|c|c|c|}
\hline & \multicolumn{2}{|c|}{ Vinyl } & \multicolumn{2}{|c|}{ Carpet } & \multicolumn{2}{|c|}{ Rubber } \\
\hline & $M$ & $S D$ & $M$ & $S D$ & $M$ & $S D$ \\
\hline Lead block slip threshold & 16.90 & 0.99 & 30.96 & 2.06 & 50.80 & 3.58 \\
\hline \multicolumn{7}{|l|}{ Experiment 1: Perceptual judgments } \\
\hline $0^{\circ}$ & 19.50 & 5.03 & 25.31 & 6.16 & 27.29 & 7.27 \\
\hline $90^{\circ}$ & 24.00 & 5.26 & 31.40 & 5.62 & 30.83 & 6.39 \\
\hline Average & 21.75 & 5.06 & 28.35 & 5.78 & 29.06 & 6.73 \\
\hline \multicolumn{7}{|l|}{ Experiment 2: Perceptual judgments } \\
\hline $0^{\circ}$ & 20.48 & 5.19 & 25.00 & 4.93 & 27.90 & 5.86 \\
\hline $90^{\circ}$ & 24.65 & 4.62 & 30.38 & 4.51 & 32.56 & 4.29 \\
\hline Average & 22.56 & 4.80 & 27.69 & 4.43 & 30.23 & 5.02 \\
\hline
\end{tabular}

Note. Means represent slant of the slope in degrees. $0^{\circ}=$ trials beginning with the slope set to $0^{\circ} ; 90^{\circ}=$ trials beginning with the slope set to $90^{\circ}$; Average $=$ judgments averaged across both slope positions .

task and with using the remote to adjust the slope. After the carpet condition, half of the participants received the low-friction vinyl trials first, and the other half received high-friction rubber trials first, with the starting position of the slope $\left(0^{\circ} / 90^{\circ}\right)$ counterbalanced.

Trials began when participants stood on the viewing platform and faced the sloping walkway. The experimenter handed the remote to the participants and said, "Adjust the slant to the steepest degree that you think you could walk down without slipping or falling. Remember, walking means taking two full steps on the slope so that both feet touch the slant at least once. Keep in mind, the surface you are standing on is exactly the same as the surface lining the slope." There was no time limit.

At the end of each trial, participants handed the remote to the experimenter, turned around, and stepped off the viewing surface while the experimenter set the slope to the starting position for the next trial. After completing four trials on each surface, participants left the laboratory while the experimenter changed the surfaces lining the sloping walkway and the viewing platform for the next condition.

\section{Results and Discussion}

Initial tests for current and all subsequent experiments showed that perceptual judgments were not affected by presentation order of low- and high-friction surfaces ( $p$ s $>.10$ ). Therefore, data were collapsed across presentation orders for further analyses. We corrected for experiment-wise error by using Bonferroni-adjusted alpha levels ( $p=.05 /$ number of tests) when more than one $t$ test was performed.

A 3 (vinyl, carpet, and rubber surface) $\times 2\left(0^{\circ}\right.$ and $90^{\circ}$ starting position) repeated measures analysis of variance (ANOVA) on perceptual judgments showed main effects for surface, $F(2,46)=$ $37.24, p<.01$, and starting position, $F(1,23)=195.40, p<.01$, and an interaction between surface and starting position, $F(2$, $46)=11.56, p<.01$ (Table 1, Experiment 1). The main effect for surface reflected participants' sensitivity to standing on lowfriction vinyl: Participants judged that they could walk down steeper slopes while standing on carpet $\left(M=28.35^{\circ}, S D=5.78\right)$ and on rubber $\left(M=29.06^{\circ}, S D=6.73\right)$ than on vinyl $(M=$ $21.75^{\circ}, S D=5.06 ; p$ s $\left.<.01\right)$. Although lead block slip thresholds suggested that participants could have walked down steepest slopes in the rubber condition, participants did not differentiate between rubber and carpet $(p>10)$. Judgments were also affected by the slope's starting position. Participants judged that they could walk down steeper slopes when trials began with the slope set to $90^{\circ}\left(M=28.74^{\circ}, S D=4.99\right)$ than when it was set to $0^{\circ}$ $\left(M=24.03^{\circ}, S D=5.66, p<.01\right)$.

To examine the interaction between surface and starting position, we calculated the difference between judgments at the two starting positions $\left(0^{\circ}\right.$ and $\left.90^{\circ}\right)$ for each surface and found that the interaction resulted from difference scores that varied across surfaces. Judgments made on vinyl $\left(M\right.$ difference $=4.50^{\circ}, S D=$ 1.84 ) and on rubber ( $M$ difference $=3.54^{\circ}, S D=2.51$ ) were closer in value across the two starting positions than judgments made on carpet $\left(M\right.$ difference $\left.=6.08^{\circ}, S D=2.32, p \mathrm{~s}<.01\right)$.

To determine how closely participants' perceptual judgments reflected the actual constraints due to friction conditions, we compared their judgments with the lead block slip thresholds for each surface (see Bar 1 in each panel of Figure 2). We confirmed with $t$ tests that for low-friction vinyl and high-friction rubber surfaces, perceptual judgments were significantly different from the lead block slip thresholds. In essence, participants overestimated their ability on low-friction vinyl $\left(M\right.$ difference $=4.85^{\circ}$, $S D=2.06), t(32)=2.98, p<.01$, but underestimated their ability to walk down slopes covered with high-friction rubber ( $M$ difference $\left.=21.74^{\circ}, S D=6.73\right), t(32)=9.60, p<.01$. For carpet, perceptual judgments and lead block slip thresholds were similar $\left(M\right.$ difference $\left.=2.61^{\circ}, S D=5.78, p>.10\right)$.

In summary, participants showed sensitivity to changes in friction conditions: They distinguished low-friction vinyl from higherfriction carpet and rubber. However, sensitivity may be insufficient to guide locomotor decisions because perceptual judgments differed from the lead block slip thresholds in the low- and high-friction conditions.

\section{Experiment 2: Generating Information About Friction Via Tactile Exploration}

Results from Experiment 1 suggested that in a walking task, adults, like infants, may have difficulty in extrapolating from flat to sloping ground based on underfoot information for friction. 


\section{A. Low-friction Condition}

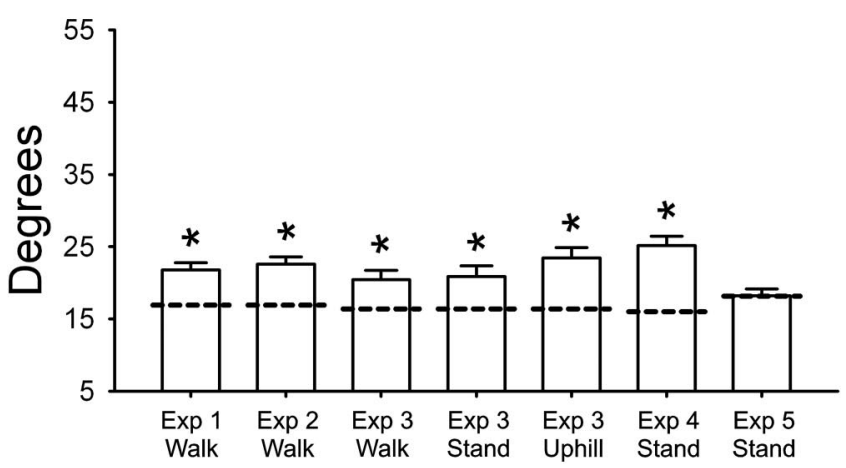

B. Medium-friction Condition

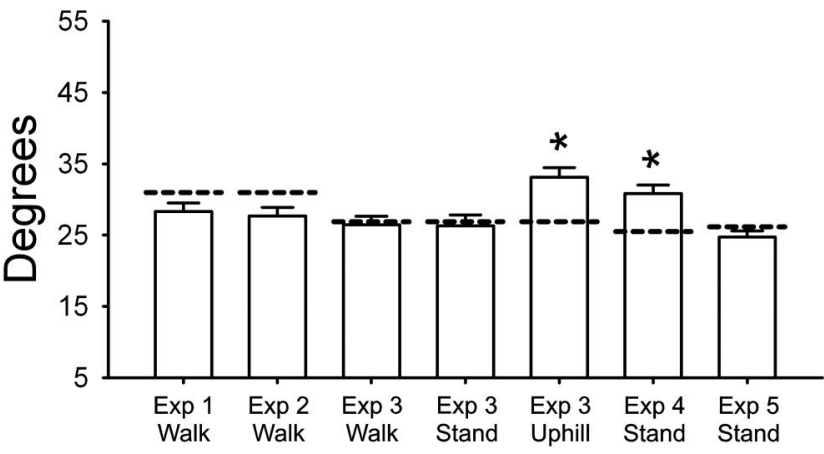

C. High-friction Condition

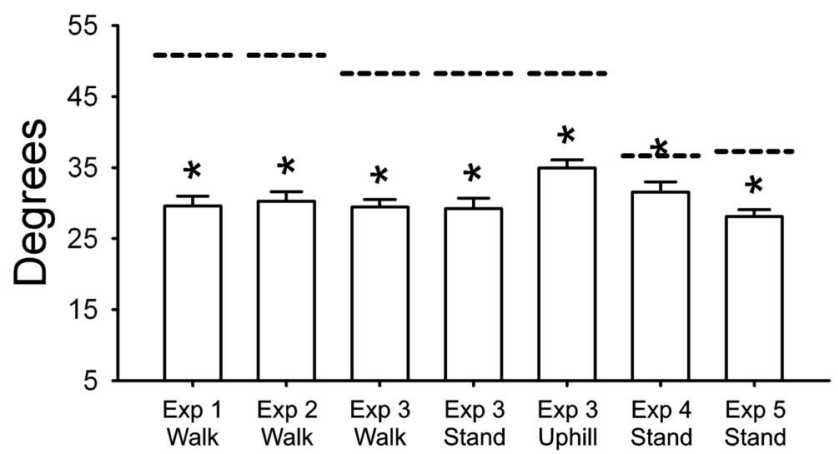

Figure 2. Mean perceptual judgments and slip thresholds for (A) lowfriction (vinyl surface or Teflon shoe sole), (B) medium-friction (carpet surface or typical shoe sole), and (C) high-friction conditions (rubber surface or rubber shoe sole) in Experiments 1-5. The vertical bars represent participants' perceptual judgments and are labeled by experiments and conditions. The horizontal dashed lines represent lead block (Experiments 1-3) and standing slip (Experiments 4-5) thresholds. Asterisks denote significant differences $(p<.05)$ between perceptual judgments and slip thresholds.

Consequently, they may attempt impossibly steep low-friction slopes and needlessly avoid safe high-friction slopes. However, analyses of the videotapes showed that participants did not engage in large, overt exploratory movements that might generate additional tactile information for adaptive locomotor decisions. In previous research (e.g., Mark et al., 1990; Warren \& Whang, 1987), small, subtle swaying and stepping movements of the head and body were sufficient to guide observers' judgments about possibilities for sitting on various chair heights and for walking through apertures of varying widths. Apparently, such subtle exploratory movements were not sufficient to produce discriminating judgments about underfoot information about friction. Thus, we considered the possibility that the perceptual judgments in Experiment 1 may have resulted from insufficient perceptual information about friction rather than an inability to incorporate underfoot information about friction into a plan for upcoming ground. Therefore, in Experiment 2, participants were instructed to perform exploratory movements that would generate additional information about friction at the start of each trial.

\section{Method}

Twenty-four adults (14 women, 10 men; $M$ age $=23.16$ years, $S D=6.63$ ) were recruited and compensated as in Experiment 1 . Two participants had experienced serious friction-related falls requiring medical care. The procedure was identical to Experiment 1 with one exception: At the start of the session, participants watched a short video of a model marching in place and "doing the twist" by sliding her feet and body from side to side. The marching movements generated frictional forces similar to the forces involved during walking, and the twisting movements guaranteed that the feet would rub vigorously against the floor. Participants practiced both movements until they could produce the full range of motions comfortably. Each trial began with six marches and six twists on the standing platform.

\section{Results and Discussion}

As in Experiment 1, an overall ANOVA showed that perceptual judgments were affected by the surface type, $F(2,46)=78.43$, $p<.01$, and starting position, $F(1,23)=121.46, p<.01$ (see Table 1, Experiment 2). The main effect for surface type resulted from participants' estimation that they could walk down steeper slopes on carpet $\left(M=27.69^{\circ}, S D=4.43\right)$ and rubber $(M=$ $\left.30.23^{\circ}, S D=5.02\right)$ than on vinyl $\left(M=22.56^{\circ}, S D=4.80, p s<\right.$ $.01)$. Participants also estimated that they could walk down steeper slopes on rubber than on carpet $(p<.01)$, suggesting that the additional perceptual information from marching and twisting movements may have increased their sensitivity to the differences in $\mathrm{COF}$ between the two higher-friction surfaces. Again, judgments were affected by the slope's starting position: $M=29.19^{\circ}$ versus $M=24.46^{\circ}(S D=4.10$ vs. $S D=4.94)$ for $90^{\circ}$ and $0^{\circ}$, respectively $(p<.01)$.

Perceptual judgments were similar across Experiments 1 and 2 ( $p$ s > .10; compare Experiments 1 and 2 in Table 1). Despite an increased sensitivity to the rubber surface, the act of generating additional tactile information for frictional forces via marching and twisting movements did not improve the overall accuracy of participants' judgments with respect to the lead block slip thresholds. As in Experiment 1, perceived abilities for walking down the vinyl slopes were higher than the lead block slip threshold ( $M$ difference $\left.=5.66^{\circ}, S D=4.80\right), t(32)=3.67, p<.01$, and perceived abilities for walking down the rubber slopes were lower than the average lead block slip threshold $\left(M\right.$ difference $=20.57^{\circ}, S D=$ 5.02), $t(32)=11.74, p<.01$. Perceptual judgments and lead block slip thresholds were similar for carpet $\left(M\right.$ difference $=3.27^{\circ}$, 
$S D=4.43, p>.10$ ). In summary, exploratory movements led to greater sensitivity between the different surfaces. However, participants did not show improvements in the match between perceptual judgments and lead block slip thresholds (see Bar 2 in each panel of Figure 2).

\section{Experiment 3: Wearing Shoes With Low- and High- Friction Soles}

Despite findings from two experiments that observers may not take underfoot information about friction into account for gauging affordances on slopes, the evidence for this conjecture was not optimal: We asked participants to make judgments about possibilities for walking downhill, but estimated lead block slip thresholds from a nonmoving, "standing" block. Possibly, the disparity between perceptual judgments and lead block slip thresholds on the vinyl and rubber surfaces reflected only a disparity between walking and standing abilities. On slopes, standing is a more stringent test of locomotor ability than walking: It may be possible for adults to walk down a small slope that is impossible for them to stand on because while walking, they can exploit rather than fight the forces pulling their bodies downward by coasting along the surface without falling.

In addition, the finding that perceptual judgments converged toward the carpet surface may reflect the familiarity of carpet and the novelty of vinyl and rubber, rather than participants' ability to use information for friction to make adaptive locomotor decisions. Previous work showed that observers erroneously base judgments about slip on visual cues such as surface color and shine (Joh, Adolph, Campbell, \& Eppler, 2006). It is possible that the different feel-but similar appearance — of the low- and high-friction surfaces affected participants' perceptual judgments.

Therefore, in Experiment 3, we controlled for visual cues for friction conditions by covering the viewing platform and slope with carpet on all trials and varied friction conditions by changing participants' shoe soles. To address the discrepancy between perceptual judgments for walking and lead block slip thresholds for standing, we asked participants to estimate the steepest slope that they could stand on as well as walk down. In addition, we asked participants to estimate the steepest slope they could walk up to test the generality of the findings to a different task.

\section{Method}

Twenty-four adults ( 15 women, 9 men; $M$ age $=21.74$ years, $S D=3.23$ ) were recruited and compensated as in the previous experiments. Four participants had experienced friction-related falls requiring medical attention. Two additional participants were excluded from the final sample due to equipment failure.

All equipment was identical to Experiments 1 and 2, except that the surface covering the viewing platform and sloping walkway was plush carpet (used as the medium-friction surface in Experiments 1 and 2). Changes in friction conditions were accomplished by dressing participants in sneakers with custom-made, removable soles that attached with Velcro. In the low-friction condition, the soles were covered with a thin sheet of Teflon. In the mediumfriction condition, the soles were a typical shoe material (crepe outsoles). In the high-friction condition, the soles were covered with the rubber Dycem used in previous experiments. Lead block slip thresholds, obtained as before, were $16.40^{\circ}(S D=1.58)$ for the Teflon sole, $26.90^{\circ}(S D=1.45)$ for the typical sole, and $48.20^{\circ}$ $(S D=1.62)$ for the rubber sole (see Table 2).

There were 36 trials total, 12 per task. The procedure was identical to Experiment 1 for the downhill walking task, except that participants' shoe soles, not the surfaces covering the slope, were changed to create the different friction conditions. For the standing task, the experimenter asked participants to gauge the steepest slope they could stand on without slipping and falling, rather than walk down. The uphill task was identical to the downhill task except that participants stood at the base of the slope, rather than the top, and "up" replaced "down" in the instructions to participants.

\section{Results and Discussion}

A 3 (judgment task) $\times 3$ (shoe sole) $\times 2$ (slope's starting position) repeated measures ANOVA showed that perception of

Table 2

Experiment 3: Lead Block Slip Thresholds and Perceptual Judgments

\begin{tabular}{|c|c|c|c|c|c|c|}
\hline & \multicolumn{2}{|c|}{ Teflon sole } & \multicolumn{2}{|c|}{ Typical sole } & \multicolumn{2}{|c|}{ Rubber sole } \\
\hline & $M$ & $S D$ & $M$ & $S D$ & $M$ & $S D$ \\
\hline Lead block slip threshold & 16.40 & 1.58 & 26.90 & 1.45 & 48.20 & 1.62 \\
\hline \multicolumn{7}{|l|}{ Downhill walking: Perceptual judgments } \\
\hline $0^{\circ}$ & 18.73 & 6.27 & 24.13 & 6.03 & 28.23 & 5.21 \\
\hline $90^{\circ}$ & 22.10 & 6.51 & 28.77 & 5.86 & 31.63 & 5.20 \\
\hline Average & 20.42 & 6.33 & 26.45 & 5.84 & 29.95 & 5.09 \\
\hline \multicolumn{7}{|l|}{ Downhill standing: Perceptual judgments } \\
\hline $0^{\circ}$ & 19.13 & 7.13 & 24.46 & 7.78 & 27.46 & 7.58 \\
\hline $90^{\circ}$ & 23.00 & 7.59 & 28.06 & 7.18 & 31.10 & 6.99 \\
\hline Average & 20.88 & 7.21 & 26.30 & 7.41 & 29.28 & 7.18 \\
\hline \multicolumn{7}{|l|}{ Uphill walking: Perceptual judgments } \\
\hline $0^{\circ}$ & 21.46 & 7.07 & 30.88 & 7.28 & 32.96 & 5.89 \\
\hline $90^{\circ}$ & 25.40 & 7.35 & 35.31 & 6.56 & 36.88 & 5.86 \\
\hline Average & 23.43 & 7.12 & 33.09 & 6.78 & 34.92 & 5.65 \\
\hline
\end{tabular}

Note. Means represent slant of the slope in degrees. $0^{\circ}=$ trials beginning with the slope set to $0^{\circ} ; 90^{\circ}=$ trials beginning with the slope set to $90^{\circ}$; Average $=$ judgments averaged across both slope positions. 
ability was affected by task, $F(2,46)=16.74, p<.01$, shoe sole, $F(2,46)=48.99, p<.01$, and slope's starting position, $F(1,23)=$ $123.15, p<.01$. We also found a Task $\times$ Shoe Sole interaction, $F(4,92)=6.28, p<.01$ (see Table 2$)$.

The main effect for task resulted from participants' perception that they could walk up steeper slopes $\left(M=30.48^{\circ}, S D=5.32\right)$ than they could walk down $\left(M=25.58^{\circ}, S D=4.97\right)$ or stand on downhill $\left(M=25.50^{\circ}, S D=6.56, p \mathrm{~s}<.01\right)$. The similarity in judgments for the two downhill tasks indicates that findings from Experiments 1 and 2 did not result from our asking participants about walking judgments and comparing them to "standing" lead block slip thresholds. The main effect for shoe sole revealed that participants were sensitive to differences in low- and high-friction conditions. Perceptual judgments were lowest for low-friction Teflon soles $\left(M=21.58^{\circ}, S D=6.27\right)$, followed by typical shoe soles $\left(M=28.62^{\circ}, S D=5.48\right)$, and then high-friction rubber soles $\left(M=31.38^{\circ}, S D=5.38, p \mathrm{~s}<.01\right)$.

The Task $\times$ Shoe Sole interaction was due to the larger difference in judgments between uphill walking and downhill walking and standing for typical and rubber soles compared to Teflon soles. We calculated difference scores between uphill walking judgments and the average of downhill walking and standing judgments. Difference scores for Teflon soles $\left(M\right.$ difference $=1.04^{\circ}, S D=$ 4.74) were significantly lower than difference scores for typical soles $\left(M\right.$ difference $\left.=3.48^{\circ}, S D=5.55, p=.01\right)$ and rubber soles $\left(M\right.$ difference $\left.=3.18^{\circ}, S D=4.38, p=.016\right)$. Difference scores for typical and rubber soles were similar $(p>.10)$. As before, the main effect for starting position was due to participants' estimating that they could navigate steeper slopes on trials starting with the slope set to $90^{\circ}\left(M=29.20^{\circ}, S D=5.94\right)$ than on those with the slope set to $0^{\circ}\left(M=25.23^{\circ}, S D=5.03, p<.01\right)$.

Overall, across tasks, perceptual judgments differed from lead block slip thresholds, providing additional evidence that the results from Experiments 1 and 2 were not caused by methodological artifacts (compare lead block slip thresholds, downhill walking, downhill standing, and uphill walking in Table 2; Bars 3-5 in each panel of Figure 2). On low-friction Teflon sole trials, participants overestimated their ability to walk downhill ( $M$ difference $=$ $\left.4.02^{\circ}, S D=6.33\right), t(32)=1.97, p=.06$, stand on downhill slopes $\left(M\right.$ difference $\left.=4.48^{\circ}, S D=7.21\right), t(32)=1.93, p=.06$, and walk uphill $\left(M\right.$ difference $\left.=7.03^{\circ}, S D=7.12\right), t(32)=3.06, p<$ .01. On high-friction rubber sole trials, compared to the lead block slip thresholds, participants underestimated their ability to walk downhill $\left(M\right.$ difference $\left.=18.25^{\circ}, S D=5.09\right), t(32)=11.027, p<$ .01 , stand on downhill slopes $\left(M\right.$ difference $\left.=18.92^{\circ}, S D=7.18\right)$, $t(32)=8.18, p<.01$, and walk uphill $\left(M\right.$ difference $=13.28^{\circ}$, $S D=5.65), t(32)=7.26, p<.01$. Participants were so confident about walking uphill that they even overestimated their ability in typical shoe soles $\left(M\right.$ difference $\left.=6.19^{\circ}, S D=6.77\right), t(32)=$ $2.84, p<.01$. Perceived abilities and lead block slip thresholds did not differ for downhill walking $\left(M\right.$ difference $\left.=0.45^{\circ}, S D=5.84\right)$ and standing judgments ( $M$ difference $=0.60^{\circ}, S D=7.41, p \mathrm{~s}>$ .10) made in typical shoe soles.

By testing participants in three different tasks and varying friction conditions with shoe soles rather than floor coverings, we replicated the previous results, extended the results to new tasks, and ruled out possible artifacts. As shown in Figure 2, participants' judgments were highly consistent across studies and tasks in Experiments 1-3. Whether the task was walking downhill, stand- ing downhill, or walking uphill on carpet, participants' judgments did not match the lead block slip thresholds in the low- and high-friction conditions. Judgments were identical for standing and walking downhill tasks and differed from lead block slip thresholds.

\section{Experiment 4: Judging and Standing on Slopes}

In Experiments 1-3, perceived abilities were compared to lead block slip thresholds. COF is not affected by object properties such as mass and surface area. However, in everyday locomotion, walkers' individual, idiosyncratic, balance control strategies may affect their slip thresholds. Some strategies may increase slip thresholds (e.g., gripping the surface with the toes can provide more traction). Other strategies may decrease slip thresholds (e.g., small foot movements and postural sway can generate destabilizing torque and pull the body off balance). Thus, people standing on a slope might yield different slip thresholds than a lead block because their feet are pliable and connected to a moving body. Differences in lead block and standing slip thresholds would provide insight regarding the effectiveness of walkers' balance control strategies.

Experiment 4 was designed to test the effects of individual balance control strategies on perceptual judgments. As in the earlier studies, participants judged the steepest slope they could stand on without slipping or falling. In addition, participants actually stood on slopes of varying slants under low-, medium-, and high-friction conditions to determine whether perceived abilities matched standing slip thresholds. If participants' judgments were similar to those of Experiments 1-3 and did not match their abilities (as measured by standing slip thresholds instead of lead block slip thresholds), then the findings would provide converging evidence that walkers over- and underestimate their abilities for coping with low- and high-friction surfaces, respectively.

Measuring participants' standing slip thresholds required a walkway that could support adults' weight. Therefore, we built a new apparatus and retained the width and length of the original slope apparatus to ensure that participants viewed and judged the same size slope across all experiments. In addition, the new walkway improved on the design of the original walkway because the standing platform was connected to the slope and level with its brink, providing a more natural eye height for judging the slope and continuous viewing and standing surfaces. We varied friction conditions by using low-friction vinyl, medium-friction carpet, and high-friction rubber surfaces. We tested standing rather than walking because it was easier to ensure participants' safety on the steep slopes.

\section{Method}

Participants and apparatus. Twenty-four adults (16 women, 8 men; $M$ age $=20.84$ years, $S D=2.13$ ) were recruited and compensated as in the previous experiments. One participant had experienced a friction-related fall requiring medical care. Participants were tested on a new walkway built to accommodate adults' height and weight. A flat, stationary standing platform $(87 \mathrm{~cm}$ wide $\times 83 \mathrm{~cm}$ long $\times 76 \mathrm{~cm}$ high) was connected to an adjustable slope $(87 \mathrm{~cm}$ wide $\times 90 \mathrm{~cm}$ long). As shown in Figure 3 , the standing platform was level with the start of the slope and provided 
A. Perceptual Judgment

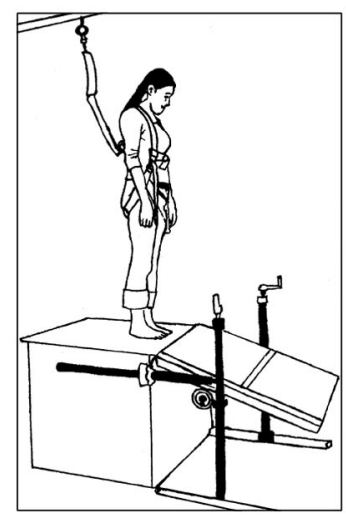

B. Standing Slip Threshold

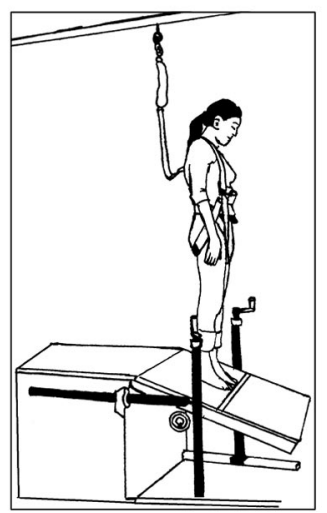

Figure 3. (A) In Experiment 4, participants first provided perceptual judgments for standing on slopes covered with high- and low-friction surfaces. (B) Next, they stood on the slope to estimate their standing slip thresholds to test the accuracy of their judgments.

a continuous surface. The slope could be adjusted in continuous increments from $0^{\circ}-50^{\circ}$ by cranking two handles simultaneously. Two cameras recorded the degree of slant indicated by the protractors placed on the underside of the slope (invisible to participants). The walkway was covered with vinyl, carpet, or rubber as in Experiments 1 and 2.

Procedure. Participants wore a full-body harness for safety, and two experimenters stood near the participants to provide assistance if necessary. As in Experiments 1 and 2, participants wore nylon knee-high tights to control for the effects of footwear on surface. The procedure for perceptual judgments was identical to Experiment 1 (see Figure 3A), except that half of the trials began with the slope at $50^{\circ}$ rather than at $90^{\circ}$. Next, we determined participants' standing slip thresholds for each surface (see Figure $3 \mathrm{~B})$ to the nearest $2^{\circ}$ or $3^{\circ}$. On the first trial, the slope was set to the average of the participant's four judgments for that surface. Participants were asked to walk carefully to the center of the slope (46 cm, marked with a line in Figure 3B) with assistance from the experimenters and to stand independently for $5 \mathrm{~s}$. If participants slipped or fell while they attempted to stand on the slope, or indicated that they could not walk down to the center of the slope without losing balance, then the slope was decreased in $5^{\circ}$ increments until participants demonstrated that they could stand on the slope without slipping. Next, the slant was increased by $3^{\circ}$ so that we could estimate participants' standing slip threshold. If participants did not slip or fall on their first trial, the slant was systematically increased in $5^{\circ}$ increments until they slipped, and then the slant was decreased by $3^{\circ}$. Trials were repeated for reliability.

\section{Results and Discussion}

Overall, results replicated the earlier findings. A 3 (carpet, rubber, and vinyl surface $) \times 2\left(0^{\circ}\right.$ and $50^{\circ}$ starting position $)$ repeated measures ANOVA confirmed that perceptual judgments were influenced by surface type, $F(2,46)=30.67, p<.01$, and starting position, $F(1,23)=151.554, p<.01$. The ANOVA also yielded a Surface $\times$ Starting Position interaction, $F(2,46)=4.02$, $p<.03$ (see Table 3, Experiment 4). As in previous studies, the main effect for surface verified that participants could differentiate between low- and high-friction surfaces. Judgments were higher on carpet $\left(M=30.84^{\circ}, S D=5.80\right)$ and rubber $\left(M=31.54^{\circ}, S D=\right.$ 7.14) compared to vinyl $\left(M=25.18^{\circ}, S D=6.21, p \mathrm{~s}<.01\right)$; judgments were similar for carpet and rubber $(p>.10)$. The main effect for starting position was due to participants' tendency to choose steeper slopes when the trials began with the slope at $50^{\circ}$ $\left(M=34.03^{\circ}, S D=5.35\right)$ than when the trials began at $0^{\circ}(M=$ $\left.24.35^{\circ}, S D=6.95, p<.01\right)$.

To examine the Surface $\times$ Starting Position interaction, we computed for each surface the difference in perceptual judgments across the two starting positions $\left(0^{\circ}\right.$ and $\left.50^{\circ}\right)$. The differences were similar for vinyl $\left(M\right.$ difference $\left.=8.85^{\circ}, S D=3.70\right)$ and rubber $(M$ difference $\left.=8.75^{\circ}, S D=3.61, p>.10\right)$. However, the difference was larger for carpet $\left(M\right.$ difference $^{\circ}=11.44, S D=6.74, p \mathrm{~s}<$ $.05)$, which shows that the interaction reflected differential effects of slope starting position on the different surfaces.

Standing slip thresholds were lowest for vinyl, followed by carpet, and then rubber $(p s<.01$; see Table 3 ). The range of standing slip thresholds was wide for carpet (range $=18^{\circ}-31^{\circ}$ ) and rubber $\left(\right.$ range $=29^{\circ}-43^{\circ}$ ), which indicates that individual's balance control strategies can help or hinder slipping. The range in thresholds was extremely narrow for vinyl (range $=14^{\circ}-18^{\circ}$ ), highlighting the difficulty of coping with low-friction slopes.

As shown in Tables 1 and 3, standing and lead block slip thresholds were nearly identical for low-friction vinyl ( $M$ difference $=.90^{\circ}$ ), but standing slip thresholds averaged $5.46^{\circ}$ lower for medium-friction carpet and $14.13^{\circ}$ lower for high-friction rubber. These findings suggest that participants' balance control strategies were ineffective on the slippery vinyl and may have hindered their ability to stand on the rubber. An additional possibility for the discrepancy on the carpet surface is that participants' heavier weight depressed the carpet pile and reduced the COF. An additional possibility for the pronounced discrepancy on the rubber surface is that there was a range of slopes preceding the slip threshold on which the lead block slipped a few centimeters and then caught again. Possibly, such small slips with human participants were sufficient to throw them off balance and cause a fall.

The most important test in Experiment 4 was the match between participants' perceptual judgments and their standing slip thresholds. We found the same pattern of over- and underestimation of ability seen in previous experiments (compare across experiments in Figure 2), although the magnitude of the discrepancy decreased with the standing slip thresholds on rubber. On the low-friction surface, the average overestimation was $9.18^{\circ}(S D=6.21, p<$ $.01)$; on the medium-friction surface, the average underestimation was $5.34^{\circ}(S D=6.50, p<.01)$; and on the high-friction surface, the average underestimation was $5.13^{\circ}(S D=5.12, p<.01)$. Discrepancies of $5^{\circ}-9^{\circ}$ have important functional implications. Increasing slant by $2^{\circ}-3^{\circ}$ caused participants to slip on surfaces that had previously afforded comfortable standing. Many slips were quite dramatic, leaving participants dangling in the harness.

We computed difference scores (perceptual judgment - standing slip threshold) for each participant for each surface and found large individual differences in the accuracy of perceived abilities. Negative difference scores denote underestimation of standing ability, and positive values denote overestimation. Difference scores ranged from $-2.25^{\circ}$ to $19.75^{\circ}$ for vinyl, $-7^{\circ}$ to $17^{\circ}$ for carpet, and $-17.75^{\circ}$ to $2.50^{\circ}$ for rubber. Only $16.7 \%, 29.2 \%$, and 
Table 3

Experiments 4 and 5: Standing Slip Thresholds and Perceptual Judgments

\begin{tabular}{|c|c|c|c|c|c|c|}
\hline & \multicolumn{2}{|c|}{ Vinyl } & \multicolumn{2}{|c|}{ Carpet } & \multicolumn{2}{|c|}{ Rubber } \\
\hline & $M$ & $S D$ & $M$ & $S D$ & $M$ & $S D$ \\
\hline Experiment 4: Standing slip threshold & 16.00 & 1.18 & 25.50 & 3.06 & 36.67 & 3.55 \\
\hline \multicolumn{7}{|l|}{ Experiment 4: Perceptual judgments } \\
\hline $0^{\circ}$ & 20.75 & 6.96 & 25.13 & 7.84 & 27.17 & 7.71 \\
\hline $50^{\circ}$ & 29.60 & 5.95 & 36.56 & 5.34 & 35.92 & 7.01 \\
\hline Average & 25.18 & 6.21 & 30.84 & 5.80 & 31.54 & 7.14 \\
\hline Experiment 5: Standing slip thresholds & 18.13 & 1.68 & 26.13 & 1.94 & 37.25 & 3.30 \\
\hline \multicolumn{7}{|l|}{ Experiment 5: Perceptual judgments } \\
\hline $0^{\circ}$ & 15.00 & 3.50 & 20.48 & 4.24 & 24.50 & 6.09 \\
\hline $50^{\circ}$ & 21.44 & 6.05 & 29.00 & 5.23 & 31.69 & 5.45 \\
\hline Average & 18.22 & 4.46 & 24.74 & 4.16 & 28.09 & 4.77 \\
\hline
\end{tabular}

Note. Means represent slant of the slope in degrees. $0^{\circ}=$ trials beginning with the slope set to $0^{\circ} ; 50^{\circ}=$ trials beginning with the slope set to $50^{\circ}$; Average $=$ judgments averaged across both slope positions .

$45.8 \%$ of participants provided perceptual judgments that fell within $\pm 3^{\circ}$ of their standing slip thresholds on vinyl, carpet, and rubber, respectively (see Figures 4A-4C). Perceptual judgments were correlated across all conditions (all $d f \mathrm{~s}=24, p \mathrm{~s}<.01$ ), showing that participants who tended to give higher perceptual judgments did so across all three surfaces.

In summary, in taking individual differences in balance control strategies into account, we replicated our initial findings: Walkers were sensitive to information about friction underfoot, but judgments were not sufficiently geared to the low-friction and mediumfriction surface to prevent slipping on slopes several degrees steeper than their thresholds. Underestimation on high-friction trials allowed participants to attempt relatively steep slopes without risk of slipping.

\section{Experiment 5: Judging and Standing on Slopes With Information About Friction and Slant}

Together, Experiments 1-4 provided converging evidence that adult walkers would be likely to err if relying on their sensitivity to friction underfoot on flat ground while gauging possibilities for coping with upcoming slopes. Without explicit instruction, participants rarely performed useful exploratory movements to obtain information about friction conditions. Even in Experiment 2, in which large, mandatory exploratory movements helped participants to differentiate between high-friction rubber and carpet, perceptual judgments did not reflect the actual potential for coping with slopes.

Thus, in our final experiment, we considered the possibility that direct contact with the slope might be required for more accurate perceptual judgments. Participants were instructed to place half of one foot onto the slope so that they could feel the effects of the friction conditions on the slope during each judgment trial. Again, to take individual differences into account, we compared perceptual judgments with standing slip thresholds.

\section{Method}

Twenty-four young adults ( 15 women, 9 men; $M$ age $=21.58$ years, $S D=3.89$ ) were recruited and compensated as in the previous experiments. Two participants reported experiencing se- rious friction-related falls. Procedures were identical to Experiment 4 with one exception: During the perceptual judgment task, participants positioned their right foot so that the back half was on the starting platform and the front half was on the slope. This placement guaranteed that the participant would obtain tactile information about friction conditions simultaneously with visual information about the slant of the slope.

\section{Results and Discussion}

A 3 (surface type) $\times 2$ (starting position) repeated measures ANOVA showed main effects for surface, $F(2,46)=78.64, p<$ .01 , and starting position, $F(1,23)=74.88, p<.01$, on perceptual judgments (see Table 3, Experiment 5). Post hoc comparisons showed that the main effect for surface was due to different judgments across all three surfaces $(p s<.01)$. Similar to Experiment 2, with additional perceptual information, participants judged that they could stand on the shallowest slope on the low-friction vinyl $\left(M=18.22^{\circ}, S D=4.46\right)$, followed by mediumfriction carpet $\left(M=24.74^{\circ}, S D=5.80\right)$, and high-friction rubber $\left(M=28.03^{\circ}, S D=4.77, p s<.01\right)$. As in previous studies, perceptual judgments were higher on trials starting with the slope set to $50^{\circ}\left(M=27.38^{\circ}, S D=4.67\right)$ compared to trials starting at $0^{\circ}\left(M=19.99^{\circ}, S D=4.08, p<.01\right)$.

We compared perceptual judgments from Experiment 5 with those of Experiments 1, 2, and 4 and found that judgments for carpet and vinyl were significantly lower in Experiment 5 ( $p$ s $<$ .02 ), perhaps because the information gleaned during direct contact with the slope made participants more cautious. Perceptual judgments for rubber did not change and remained similar across Experiments 1, 2, 4, and 5 ( $p \mathrm{~s}>.05$ ), perhaps because participants were less familiar with the material. (Experiment 3 was excluded from this comparison because the different friction conditions were achieved by carpet-shoe sole pairings rather than nylonsurface pairings.)

Overall, we replicated the standing slip threshold results from Experiment 4 (see Table 3). Across Experiments 4 and 5, standing slip thresholds were similar for carpet and rubber trials ( $p$ s $>.10$ ). On vinyl trials, participants in Experiment 5 were able to stand on steeper slopes than the participants in Experiment $4(p<.01)$. 


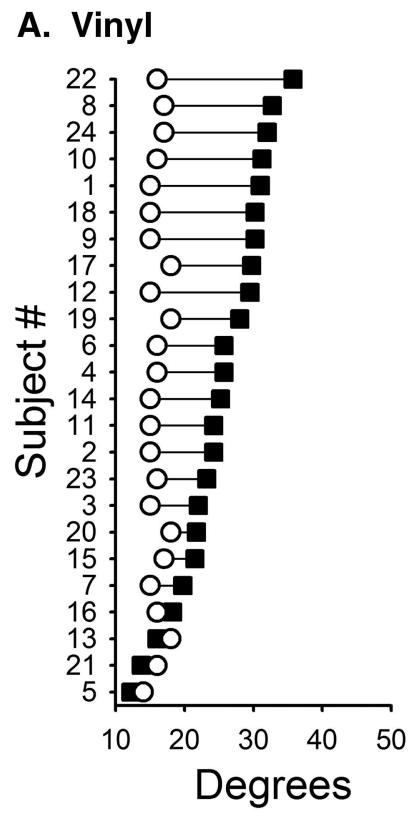

A. Vinyl

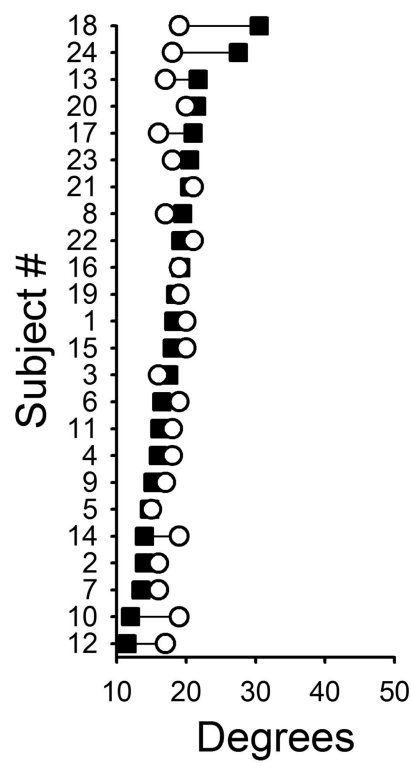

\section{Experiment 4}

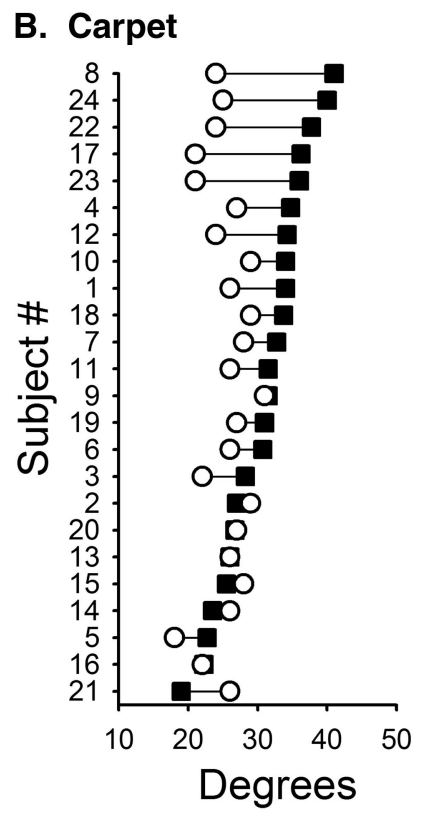

\section{Experiment 5}

\section{B. Carpet}

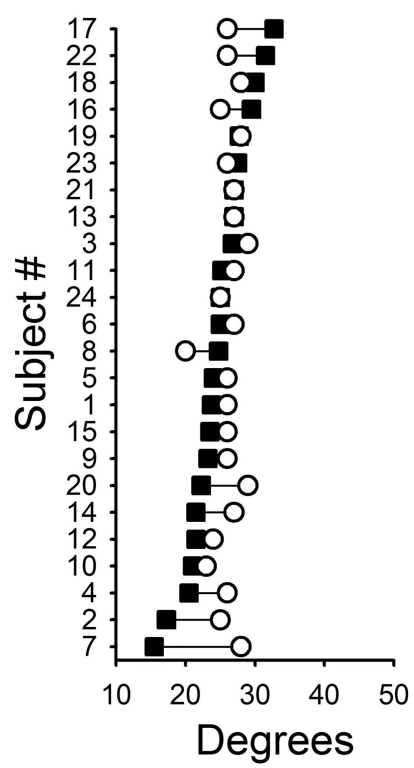

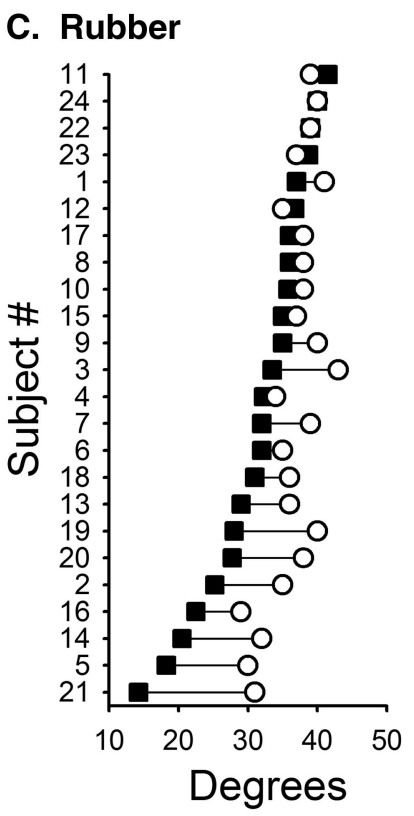

C. Rubber

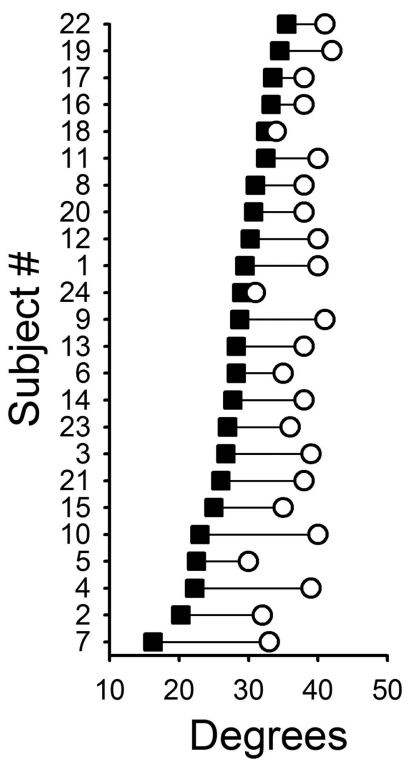

Figure 4. Individual participant's perceptual judgments and standing slip thresholds for (A and D) vinyl, (B and E) carpet, and (C and F) rubber surfaces in Experiments 4 and 5. Filled squares represent perceptual judgments and open circles represent standing slip thresholds. Slip thresholds to the left of perceptual judgments signal an overestimation of ability; slip thresholds to the right of perceptual judgments signal an underestimation of ability. For each surface, participants are ordered by their perceptual judgments and the y-axis is labeled with actual subject numbers.

Although the difference was statistically significant, it was small ( $M$ difference $=2.13^{\circ}$ ). The range of standing slip thresholds was widest for rubber $\left(\right.$ range $=30^{\circ}-42^{\circ}$ ), followed by carpet $($ range $=$ $20^{\circ}-29^{\circ}$ ), and then vinyl (range $=15^{\circ}-21^{\circ}$ ).
Comparison of participants' perceptual judgments and standing slip thresholds showed that placing just half of a foot on the slope during the trial resulted in significant improvements in perceptual judgments (compare Experiments 4 and 5 in Table 3 and Bar 7 
against Bars 1-6 in each panel of Figure 2). Perceptual judgments for both vinyl $\left(M\right.$ difference $\left.=0.09^{\circ}, S D=4.32\right)$ and carpet $(M$ difference $=1.39^{\circ}, S D=4.41$ ) were nearly identical to the respective standing slip thresholds $(p s>.10)$. In contrast, participants did not show improved judgments for rubber. They continued to underestimate their standing ability as in previous experiments $\left(M\right.$ difference $\left.=9.16^{\circ}, S D=4.18, p<.01\right)$. Although participants' perceptual judgments demonstrated that they were able to distinguish between the three surfaces, they appeared hesitant to give steep judgments for rubber.

Individual difference scores verified the improvements in the match between judgments and ability for carpet and vinyl trials and a general mismatch for rubber trials (see Figures 4D-4F). Although difference scores ranged from $-7^{\circ}$ to $11.50^{\circ}$ for vinyl and $12.50^{\circ}$ to $6.75^{\circ}$ for carpet, $70.8 \%$ and $62.5 \%$ of participants, respectively, provided perceptual judgments that fell within $\pm 3^{\circ}$ of their standing slip thresholds. Difference scores ranged from $-17^{\circ}$ to $-1.50^{\circ}$ for rubber, showing that all participants underestimated their ability on high-friction slopes. Only $8.3 \%$ of participants gave judgments that fell within $\pm 3^{\circ}$ of their rubber standing slip thresholds. Perceptual judgments were correlated across all three conditions (all $d f \mathrm{~s}=24, p \mathrm{~s}<.02$ ).

Experiment 5 showed that one way to improve participants' ability to gauge possibilities for balance on slippery slopes is to allow them to obtain tactile information about friction and slant through direct contact with the slope in addition to visual information about the slope. However, even information from direct contact was not enough to improve judgments on high-friction rubber, suggesting that walkers' familiarity with various types of surfaces may play a role in perceiving affordances for actions on them.

\section{General Discussion}

\section{Friction Underfoot Is a Poor Source of Information for Prospective Control}

Prospective control of future actions relies on perceptual information arising from ongoing movements. Underfoot information about friction is a ubiquitous source of information-every movement of the body against a surface creates resistive frictional forces - but it is rarely studied. Therefore, in five experiments, we examined whether walkers use tactile information about underfoot friction to plan their future actions. All five experiments showed that walkers are sensitive to the frictional forces under their feet: Perceptual judgments leaned in the correct direction of the slip thresholds. Observers perceived that they could navigate steeper slopes on higher-friction trials than on lower-friction trials. However, sensitivity did not ensure adaptive locomotor decisions. Participants missed the mark in terms of using current frictional forces as sources of information for action possibilities for upcoming ground. In Experiments 1-3, compared to the lead block slip thresholds, participants tended to overestimate their ability to walk and stand under low-friction conditions and underestimate their ability to walk and stand under high-friction conditions. In Experiment 4, participants even overestimated their ability to stand on carpet; on all surfaces, their perceived abilities deviated from their standing slip thresholds by at least $5^{\circ}$. Functionally, even a $2^{\circ}-3^{\circ}$ change in slant induced a fall, which shows that if participants had planned their actions based on perceptual judgments, they most likely would have slipped on the slope. Only in Experiment 5, in which participants' feet were in direct contact with the slope during each trial, did their judgments become more accurate. Similarly, infants are less likely to err if they engage in spontaneous tactile exploration of the slope by making direct contact with their feet at the brink (Adolph et al., 2007). However, adults' judgments improved for carpet and vinyl only and did not improve for rubber.

Not only did the feeling of slip underfoot fail to focus walkers' attention on friction conditions as a basis for guiding future action, but changing friction conditions also did not prompt walkers to engage in concerted tactile exploration. Exploratory movements are necessary for gathering perceptual information for prospective control. Previous studies (Adolph et al., 2007; Lo et al., 1999; Mark et al., 1990; Warren \& Whang, 1987) showed that exploratory stepping, swaying, rocking, and shuffling movements improve perceptual judgments in infant and adult walkers. Indeed, in Experiment 2, participants showed increased sensitivity and discrimination of surfaces after performing mandated twisting and marching movements. However, in other experiments, without explicit instructions to explore, participants rarely produced such movements on their own. Instead, most stood so still that exploratory movements could not be discerned reliably from the videotapes.

Failure to base judgments on information about friction is not limited to laboratory studies. Outside the lab, failure to perceive friction conditions can prevent walkers from planning their future actions appropriately and can have dire consequences for safety. According to recent national statistics, falling is the most common cause of unintentional, nonfatal injuries and the second most common cause of fatal injuries in the United States (National Safety Council, 2003). Many falls are friction-related, resulting from slipping on low-friction ground. Slip-related accidents account for $64 \%$ of falls in the workplace and $25 \%$ in the home (Courtney, Sorock, Manning, Collins, \& Holbein-Jenny, 2001; National Safety Council, 2003). Indeed, across the five experiments in the current study, $8 \%$ of healthy young adults reported a friction-related slip that required medical attention. Moreover, slipping and falling incidents are likely to be underreported (Strandberg, 1983). Fall-related deaths and injuries may be attributed to other causes. These statistics explain why researchers in ergonomics and safety sciences have dedicated vast amounts of time and resources to building shoes and floors safer for walking under varying conditions, creating home and work environments that reduce slipping and falling, designing effective warning signs for dangerous risky ground, and so on (e.g., Chiou, Bhattacharya, \& Succop, 1996; Leclercq, 1999; Myung \& Smith, 1997; Patla, 1997a; Swensen, Purswell, Schlegel, \& Stanevich, 1992).

\section{Why Do Walkers Disregard Information for Friction?}

Why do frictional forces appear to play such a minor role in prospective control of balance and locomotion? Why might walkers disregard the feeling of slip underfoot in favor of visual information? Why might changes in friction conditions fail even to elicit information-seeking exploratory movements for prospective control with regard to upcoming events?

One possibility is that participants' judgments resulted from a systematic failure in slope perception, not an inability to incorpo- 
rate information about friction into a plan for action. For example, Proffitt et al. (1995) asked college students to estimate the incline of $2^{\circ}-60^{\circ}$ hills using verbal, visual, and haptic tasks. Hills were presented in real or virtual environments, and participants were asked to make judgments from the top and the bottom of the hill. Across all conditions, when using verbal and visual measures, participants overestimated the slant of the hill by $8^{\circ}$ (for the shallowest hills) to $20^{\circ}$ (for steeper hills). Haptic judgments were accurate.

There are, however, two important differences between the previous data and ours. First, our participants made judgments about action possibilities whereas participants in the Proffitt et al. (1995) study made judgments about slant. Second, our participants showed both over- and underestimation of slopes. On high-friction trials, as in the Proffitt et al. study, participants underestimated their abilities, suggesting an overestimation of slope. But on low-friction trials, participants overestimated their abilities, suggesting an underestimation of slant. These differences suggest that our findings may not have resulted (solely) from misperceptions of slant.

A more likely possibility is that friction is simply too complex to be a useful predictor. The magnitude of the frictional forces that emerge when two surfaces come into contact depends on an unbounded set of factors that interact in complex and nonlinear ways. The manner in which the two surfaces touch (e.g., angle of the walker's body), changes to one or both of the surfaces from repeated contact (e.g., tears on shoe soles from repeated wear), and moment to moment variations in the context (e.g., condensation on the ground due to humidity) are only a few factors that can cause unexpected, nonlinear changes in friction conditions. As a testament to the complexity of friction, researchers fiercely disagree on the most accurate method for measuring COF (Chang et al., 2001; Leclercq, 1999). Dozens of slip-meter devices have been proposed to capture COF (Strandberg, 1985). Researchers have no principled rationale for determining a safe $\mathrm{COF}$ level, an optimal shoe sole, or an optimal ground surface for work environments. They can only suggest reasonable rules of thumb.

Like researchers, walkers may learn to adhere to general rules of thumb when coping with slippery surfaces rather than attempting precise prediction based on additional exploration. For example, walkers may downsize their expected abilities for upcoming ground when they feel their feet slip and scale up their expectations when they feel their feet grip but not bother to determine exact slip levels. Explicit rules of thumb, learned from past experiences, rather than on-line perceptual information, may prompt us to wear more gripping shoes for a hike, icy walk, or sport that requires good push-off and to lay carpets over wet entryways, line our bathtubs with rubber mats, and sprinkle salt on our sidewalks. Familiarity with carpet and slippery surfaces also may have helped to improve participants' judgments in Experiment 5, while at the same time their lack of familiarity with rubber Dycem resulted in no changes in judgments.

If tactile, underfoot information about friction is too complex to allow accurate prospective control of locomotion, a related possibility is that walkers adopt rules of thumb that are based on unreliable sources of visual information for friction to determine action possibilities. For example, a recent study found that respondents rely on shine to predict whether upcoming ground is slippery (Joh et al., 2006). Although visual ratings of shine and slip are moderately correlated, both judgments are affected by surface color, viewing distance, and lighting conditions-factors that do not affect the actual COFs. Participants may learn to use visual cues such as shine to predict slip because the complicated nature of friction leaves them few viable options or because their prior experiences lead to beliefs that influence their judgments.

\section{What Is a Walker to Do?}

Given the complexity of underfoot friction and the unreliability of visual cues for friction, what is a walker to do to avoid friction-related falls? The most common choice may be to exercise friction-based prospective control on a limited local basis. Feedback from the last step elicits gait modifications for the next step: Indeed, after one step on a slippery surface, both infant and adult walkers take smaller, slower, more flat-footed steps (Cham \& Redfern, 2002; Joh \& Adolph, 2007; Marigold \& Patla, 2002; Myung \& Smith, 1997; Patla, 1997a; Swensen et al., 1992; You et al., 2001). But such reactive strategies are inefficient; locomotion requires prospective control. Therefore, when a walker is forming a plan that incorporates variations in the layout of the terrain (e.g., slopes), information about friction may recede to the background and reliance on visual information for slant may come to the fore. Participants' overly cautious responses to the high-friction rubber surface- - despite the fact that the high-friction surface would allow them to safely cope with steep slopes - suggests a rule of thumb for relying on information about slant rather than friction conditions, especially on unfamiliar surfaces. However, when the ground surface itself is variable, such as when walkers approach a patch of ice, reliance on visual cues such as shine is likely to lead to errors.

Another choice is to form context-specific associations about particular visual cues and consequences for locomotion. Adultsand to some extent, infants and children-can learn to link the arbitrary cues signaling changes in friction and rigidity (e.g., color or pattern of ground) with the consequence of falling (Joh \& Adolph, 2006, 2007). Like friction, rigidity is a resistive force that emerges as two surfaces come into contact with each other, and novel variations are not specified via reliable visual cues from a distance. These findings suggest that adults are likely to learn associations between arbitrary visual cues for changes in friction with slipping and falling and to use the associations for prospective control. However, this strategy is also limited in its usefulness. Association learning is highly contextualized and requires at least one, potentially dangerous, error to have occurred in the past for learning to take place.

Instead, the current experiments suggest that a more prudent strategy for prospective control would be to increase exploratory activity. Tactile information increases discrimination and predictive reliability of information for friction. Additional concerted exploration would be especially important for a change in action plan, determining the frictional forces on sloping ground, gauging slip when the ground surface is variable, and for observers who can least afford a slip or fall (e.g., the elderly and infirm).

\section{References}

Adolph, K. E. (1995). A psychophysical assessment of toddlers' ability to cope with slopes. Journal of Experimental Psychology: Human Perception and Performance, 21, 734-750.

Adolph, K. E., \& Avolio, A. M. (2000). Walking infants adapt locomotion 
to changing body dimensions. Journal of Experimental Psychology: Human Perception and Performance, 26, 1148-1166.

Adolph, K. E., Eppler, M. A., \& Gibson, E. J. (1993). Crawling versus walking infants' perception of affordances for locomotion over sloping surfaces. Child Development, 64, 1158-1174.

Adolph, K. E., Eppler, M. A., \& Joh, A. S. (2007). Infants' perception of affordances of slopes under high and low friction conditions. Manuscript submitted for publication.

Cham, R., \& Redfern, M. S. (2002). Changes in gait when anticipating slippery floors. Gait and Posture, 15, 159-171.

Chang, W.-R., Gronqvist, R., Leclercq, S., Myung, R., Makkonen, L., Strandberg, L. et al. (2001). The role of friction in the measurement of slipperiness. Part 1: Friction mechanisms and definition of test conditions. Ergonomics, 44, 1217-1232.

Chiou, S., Bhattacharya, A., \& Succop, P. A. (1996). Effect of workers' shoe wear on objective and subjective assessment of slipperiness. American Industrial Hygiene Association Journal, 57, 825-831.

Cohen, H. H., \& Cohen, D. M. (1994a). Perceptions of walking surface slipperiness under realistic conditions, utilizing a slipperiness rating scale. Journal of Safety Research, 25, 27-31.

Cohen, H. H., \& Cohen, D. M. (1994b). Psychophysical assessment of the perceived slipperiness of floor tile surfaces in a laboratory setting. Journal of Safety Research, 25, 19-26.

Courtney, T. K., Sorock, G. S., Manning, D. P., Collins, J. W., \& HolbeinJenny, M. A. (2001). Occupational slip, trip, and fall-related injuriescan the contribution of slipperiness be isolated? Ergonomics, 44, 1118 1137.

Eppler, M. A., Adolph, K. E., \& Weiner, T. (1996). The developmental relationship between infants' exploration and action on slanted surfaces. Infant Behavior and Development, 19, 259-264.

Fajen, B. R., \& Warren, W. H. (2003). Behavioral dynamics of steering, obstacle avoidance, and route selection. Journal of Experimental Psychology: Human Perception and Performance, 29, 343-362.

Fitzpatrick, P., Carello, C., Schmidt, R. C., \& Corey, D. (1994). Haptic and visual perception of an affordance for upright posture. Ecological Psychology, 6, 265-287.

Gibson, E. J., \& Pick, A. D. (2000). An ecological approach to perceptual learning and development. New York: Oxford University Press.

Gibson, J. J. (1979). The ecological approach to visual perception. Boston: Houghton Mifflin.

Joh, A. S., \& Adolph, K. E. (2006). Learning from falling. Child Development, 77, 89-102.

Joh, A. S., \& Adolph, K. E. (2007). Learning from slipping and falling. Manuscript in preparation.

Joh, A. S., Adolph, K. E., Campbell, M. R., \& Eppler, M. A. (2006). Why walkers slip: Shine is not a reliable cue for slippery ground. Perception \& Psychophysics, 68, 339-352.

Kinsella-Shaw, J. M., Shaw, R., \& Turvey, M. T. (1992). Perceiving "walk-on-able" slopes. Ecological Psychology, 4, 223-239.

Leclercq, S. (1999). The prevention of slipping accidents: A review and discussion of work related to the methodology of measuring slip resistance. Safety Science, 31, 95-125.

Lee, D. N., \& Aronson, E. (1974). Visual proprioceptive control of standing in human infants. Perception \& Psychophysics, 15, 529-532.

Lee, D. N., Lishman, J. R., \& Thomson, J. A. (1982). Visual regulation of gait in long jumping. Journal of Experimental Psychology: Human Perception and Performance, 8, 448-459.

Lo, T., Avolio, A. M., Massop, S. A., \& Adolph, K. E. (1999). Why toddlers don't perceive risky ground based on surface friction. In M. A. Grealy \& J. A. Thompson (Eds.), Studies in perception and action $v$ (pp. 231-235). Mahwah, NJ: Lawrence Erlbaum Associates.

Marigold, D. S., \& Patla, A. E. (2002). Strategies for dynamic stability during locomotion on a slippery surface: Effects of prior experience and knowledge. Journal of Neurophysiology, 88, 339-353.

Mark, L. S., Baillet, J. A., Craver, K. D., Douglas, S. D., \& Fox, T. (1990). What an actor must do in order to perceive the affordance for sitting. Ecological Psychology, 2, 325-366.

Mark, L. S., Jiang, Y., King, S. S., \& Paasche, J. (1999). The impact of visual exploration on judgments of whether a gap is crossable. Journal of Experimental Psychology: Human Perception and Performance, 25, 287-295.

Myung, R., \& Smith, J. L. (1997). The effect of load carrying and floor contaminants on slip and fall parameters. Ergonomics, 40, 235-246.

National Safety Council. (2003). Injury facts 2003 edition. Itasca, IL: Author.

Patla, A. E. (1989). In search of laws for the visual control of locomotion. Journal of Experimental Psychology: Human Perception and Performance, $15,624-628$.

Patla, A. E. (1991). Visual control of human locomotion. In A. E. Patla (Ed.), Adaptability of human gait (pp. 55-97). Amsterdam: NorthHolland/Elsevier.

Patla, A. E. (1997a). Slips, trips and falls: Implications for rehabilitation and ergonomics. In S. Kumar (Ed.), Perspectives in rehabilitation ergonomics (pp. 196-209). London: Taylor \& Francis.

Patla, A. E. (1997b). Understanding the role of vision in the control of human locomotion. Gait and Posture, 5, 54-69.

Patla, A. E. (1998). How is human gait controlled by vision? Ecological Psychology, 10, 287-302.

Patla, A. E., Prentice, S. D., Robinson, C., \& Neufeld, J. (1991). Visual control of locomotion: Strategies for changing direction and for going over obstacles. Journal of Experimental Psychology: Human Perception and Performance, 17, 603-634.

Patla, A. E., Robinson, C., Samways, M., \& Armstrong, C. J. (1989). Visual control of step length during overground locomotion: Taskspecific modulation of the locomotor synergy. Journal of Experimental Psychology: Human Perception and Performance, 15, 603-617.

Proffitt, D. R., Bhalla, M., Gossweiler, R., \& Midgett, T. (1995). Perceiving geographical slant. Psychonomic Bulletin and Review, 2, 409-428.

Proffitt, D. R., Creem, S. H., \& Zosh, W. D. (2001). Seeing mountains in mole hills: Geographical-slant perception. Psychological Science, 12, 418-423.

Stoffregen, T. A. (1985). Flow structure versus retinal location in the optical control of stance. Journal of Experimental Psychology: Human Perception and Performance, 11, 554-565.

Strandberg, L. (1983). On accident analysis and slip-resistance measurement. Ergonomics, 26, 11-32.

Strandberg, L. (1985). The effect of conditions underfoot on falling and overexertion accidents. Ergonomics, 28, 131-147.

Swensen, E. E., Purswell, J. L., Schlegel, R. E., \& Stanevich, R. L. (1992). Coefficient of friction and subjective assessment of slippery work surfaces. Human Factors, 34, 67-77.

Warren, W. H. (1984). Perceiving affordances: Visual guidance of stair climbing. Journal of Experimental Psychology: Human Perception and Performance, 10, 683-703.

Warren, W. H., \& Whang, S. (1987). Visual guidance of walking through apertures: Body-scaled information for affordances. Journal of Experimental Psychology: Human Perception and Performance, 13, 371-383.

Warren, W. H., Young, D. S., \& Lee, D. N. (1986). Visual control of step length during running over irregular terrain. Journal of Experimental Psychology: Human Perception and Performance, 12, 259-266.

You, J. Y., Chou, Y. L., Lin, C. J., \& Su, F. C. (2001). Effect of slip on movement of body center of mass relative to base of support. Clinical Biomechanics, 16, 167-173.

Received April 19, 2005

Revision received January 11, 2007 Accepted January 18, 2007 